\title{
Relation between Fisher measures of information coming from pair distribution functions
}

\author{
Á. Nagy \\ Department of Theoretical Physics, University of Debrecen, H-4010 Debrecen, Hungary \\ E. Romera \\ Departamento de Física Atómica, Molecular y Nuclear and \\ Ins tituto Carlos I de Física Teórica y Computacional, \\ Universidad de Granada, Fuentenueva s/n, $180^{` 11}$ Granada, Spain
}

(Dated: March 18, 2010)

\begin{abstract}
A relationship between three measures of Fisher information constructed from generalized pair density functions is presented. A connection to the kinetic energy is emphasized and illustrated by the Moshinsky model.
\end{abstract}




\section{INTRODUCTION}

Fisher information [1] has proved to be a very useful tool in analyzing atoms and molecules [2-21]. Previous studies generally use the electron density as a distribution function for constructing the Fisher information. Here we point out that Fisher information coming from generalized pair density functions can also be very useful. Moreover, following Koga [22], it is shown that there exist a rigorous equality between measures of Fisher information constructed from generalized pair density functions.

After an intoductory section for the Fisher information, Section 3 presents the generalized pair density functions and the relation between measures of Fisher information. Results are illustrated by the Moshinsky model in Section 4. The relationship between the kinetic energy and the Fisher information is emphasized in the last section.

\section{FISHER INFORMATION}

Fisher information [1] is a measure of the ability to estimate a parameter and is a measure of the state of disorder of a system or phenomenon. The Fisher informational functional [1] is defined as

$$
I_{f}(\theta)=\int f(x \mid \theta)\left[\frac{\partial \ln f(x \mid \theta)}{\partial \theta}\right]^{2} d x=\int \frac{\left[f^{\prime}(x \mid \theta)\right]^{2}}{f(x \mid \theta)} d x .
$$

$f(x \mid \theta)$ is a probability density function, obeying proper regularity conditions and depending on a parameter $\theta$. Take $\theta$ to be a parameter of locality:

$$
f(x \mid \theta)=f(x+\theta)=f(\gamma)
$$

Then

$$
\frac{\partial f(x \mid \theta)}{\partial \theta}=\frac{\partial f(x+\theta)}{\partial(x+\theta)}=\frac{\partial f(\gamma)}{\partial \gamma}
$$

In this case Eq. (1) has the form

$$
I_{f}(\theta)=\int\left[\frac{\partial f(x+\theta)}{\partial(x+\theta)}\right]^{2} / f(x+\theta) d x .
$$

This is Fisher information per observation with respect to the locality parameter $\theta$. As the expression does not depend on $\theta$, we may set the locality at zero:

$$
I_{f}(\theta=0)=\int \frac{\left[f^{\prime}(x)\right]^{2}}{f(x)} d x
$$


Here, we consider this case and there will be no dependence on $\theta$. Fisher information for locality is also called intrinsic accuracy. It measures the 'narrowness' of the distribution function $f$. There exist an uncertainty relation [24]

$$
I_{f} \leq 4 \sigma_{f}
$$

where $\sigma_{f}$ is the variance of the density $f$.

In this paper we establish a relationship between three measures of Fisher information. These three measures are constructed from generalized pair density functions. The following section presents a summary of these functions and presents the relation.

\section{GENERALIZED PAIR FUNCTIONS AND FISHER INFORMATION}

Koga [22] defined generalized pair density functions and derived important relations for them. We utilize these to obtain a relationship between three measures of Fisher information.

Consider an $N$-particle $\left(N \geq 2\right.$ ) amplitude (or wave) function $\Psi\left(\mathbf{r}_{1}, \mathbf{r}_{2}, \ldots, \mathbf{r}_{N}\right)$. The generalized pair density functions $g(q ; a, b)$ are defined by [22]

$$
g(q ; a, b)=\frac{2}{N(N-1)} \frac{1}{4 \pi q^{2}}\left\langle\sum_{i=1}^{N-1} \sum_{j=i+1}^{N} \delta\left(q-\left|a \mathbf{r}_{i}+b \mathbf{r}_{j}\right|\right)\right\rangle
$$

where the angular brakets stand for the expectation value over $\Psi . \delta(x)$ is the Dirac delta function. $a$ and $b$ are real-valued parameters. In this paper we normalize $g(q ; a, b)$ to 1 , while Koga had generalized pair density functions normalized to the number of particle pairs $N(N-1) / 2$. Expression (7) connects several important functions, such as the single electron density $\varrho(r)$, the pair intracule (relative motion) density $h(u)$ and the pair extracule density $d(R)$, namely

$$
\begin{gathered}
g(q ; 1,-1)=\frac{2}{N(N-1)} h(q), \\
g(q ; 1,0)=\frac{1}{N} \varrho(q)
\end{gathered}
$$

and

$$
g(q ; 1,1)=\frac{1}{4 N(N-1)} d\left(\frac{q}{2}\right) .
$$


Consider now the wave function $\Phi\left(\mathbf{p}_{1}, \mathbf{p}_{2}, \ldots, \mathbf{p}_{N}\right)$ defined in the conjugate momentum space by the Fourier transform of the position space function $\Psi\left(\mathbf{r}_{1}, \mathbf{r}_{2}, \ldots, \mathbf{r}_{N}\right)$. The generalized momentum space pair density functions $\bar{g}(q ; a, b)$ are defined by [22]

$$
\bar{g}(t ; a, b)=\frac{2}{N(N-1)} \frac{1}{4 \pi t^{2}}\left\langle\sum_{i=1}^{N-1} \sum_{j=i+1}^{N} \delta\left(t-\left|a \mathbf{p}_{i}+b \mathbf{p}_{j}\right|\right)\right\rangle .
$$

Define also a function $\bar{\chi}(t ; a, b)$ as

$$
|\bar{\chi}(t ; a, b)|^{2}=\bar{g}(t ; a, b) .
$$

Note that $\bar{\chi}(t ; a, b)$ can always be multiplied by a phase factor. Let $\nu(q ; a, b)$ denote the Fourier transform of $\bar{\chi}(t ; a, b)$ and $f(q ; a, b)$ be the distribution function corresponding to $\nu(q ; a, b)$, namely, $f(q ; a, b)=|\nu(q ; a, b)|^{2}$. The Fisher information for the distribution function $f(q ; a, b)$ is

$$
I_{f(q ; a, b)}=4 \pi \int \frac{\left[\frac{d f(q ; a, b)}{d q}\right]^{2}}{f(q ; a, b)} q^{2} d q .
$$

note that this definition agrees with definition (15) to apply relation(14). We can immediatelly notice that $I_{f(q ; a, b)}$ can be expressed with the second moment of the functions $\bar{g}(q ; a, b)$ as

$$
I_{f(q ; a, b)} \leq 4\left\langle t^{2}\right\rangle_{(a, b)}
$$

(above inequality is an equality when $\left\langle\frac{d}{d t}(\arg \chi)\right\rangle=0$, see $\left.[7,15,23]\right)$, where

$$
\left\langle t^{n}\right\rangle_{(a, b)}=4 \pi \int_{0}^{\infty} d t t^{n+2} \bar{g}(t ; a, b)=\frac{2}{N(N-1)}\left\langle\sum_{i=1}^{N-1} \sum_{j=i+1}^{N}\left|a \mathbf{p}_{i}+b \mathbf{p}_{j}\right|^{n}\right\rangle .
$$

Eq. (15) can be proved by expressing $f(q ; a, b)$ with $\bar{g}(q ; a, b)$ through the Fourier transform of $\nu(q ; a, b)$.

Koga [22] derived an important relation for the second moments of $\bar{g}(q ; a, b)$ in momentum space:

$$
\left\langle t^{2}\right\rangle_{(a, b)}+\left\langle t^{2}\right\rangle_{(a,-b)}=2\left(a^{2}+b^{2}\right)\left\langle t^{2}\right\rangle_{(1,0)}
$$

Combining Eqs. (14) and (16) we are led to

$$
I_{f(q ; a, b)}+I_{f(q ; a,-b)} \leq 2\left(a^{2}+b^{2}\right)\left\langle t^{2}\right\rangle_{(1,0)},
$$


and

$$
\left\langle t^{2}\right\rangle_{(a, b)}+\left\langle t^{2}\right\rangle_{(a,-b)} \geq \frac{1}{2}\left(a^{2}+b^{2}\right) I_{f(q, 1,0)}
$$

When $\left\langle(\arg \chi)^{\prime}\right\rangle=0$ we are led to

$$
I_{f(q ; a, b)}+I_{f(q ; a,-b)}=2\left(a^{2}+b^{2}\right) I_{f(q, 1,0)},
$$

Fisher information verifies the so called Cramer-Rao inequality for a $D$-dimensional distribution. (In our case $D=3$ ):

$$
I_{\bar{g}(q ; a, b)} \geq 9 /\left\langle t^{2}\right\rangle_{(a, b)}
$$

so taking into account Eqs.(16) and (20):

$$
I_{\bar{g}(t, 0,1)} \geq 9 \frac{2\left(a^{2}+b^{2}\right)}{\left\langle t^{2}\right\rangle_{(a, b)}+\left\langle t^{2}\right\rangle_{(a,-b)}}
$$

and

$$
2\left(a^{2}+b^{2}\right)\left\langle t^{2}\right\rangle_{(1,0)} \geq 9\left(\frac{1}{I_{\bar{g}(t, a, b)}}+\frac{1}{I_{\bar{g}(t, a,-b)}}\right)
$$

Note that for gaussians Eqs. (20), (21) and (22) are equalities and

$$
I_{\bar{g}(t ; a, b)}^{-1}+I_{\bar{g}(t ; a,-b)}^{-1}=2\left(a^{2}+b^{2}\right) I_{\bar{g}(t, 1,0)}^{-1} .
$$

Eq. (19) presents the main result of this paper. It establishes a relationship between three measures of Fisher information coming from two-parameter generalized pair distribution functions.

\section{ILLUSTRATIVE EXAMPLE}

Consider the Moshinsky model of two electrons with antiparallel spins interacting harmonically in isotropic harmonic confinement as an example. The Hamiltonian has the form

$$
H=\frac{1}{2}\left(-\nabla_{1}^{2}+r_{1}^{2}\right)+\frac{1}{2}\left(-\nabla_{2}^{2}+r_{2}^{2}\right)+\frac{1}{2} K r_{12}^{2},
$$

where

$$
\mathbf{r}_{12}=\mathbf{r}_{1}-\mathbf{r}_{2}
$$


and $K$ is the coupling constant. The ground-state position space and momentum space wave functions are

$$
\Psi\left(\mathbf{r}_{1}, \mathbf{r}_{2}\right)=C_{r} \exp \left[-\left(r_{1}^{2}+r_{2}^{2}\right) / 2-c_{r} r_{12}^{2} / 4\right]
$$

and

$$
\Phi\left(\mathbf{p}_{1}, \mathbf{p}_{2}\right)=C_{p} \exp \left[-c_{p 1}\left(p_{1}^{2}+p_{2}^{2}\right)-c_{p 2} \mathbf{p}_{1} \cdot \mathbf{p}_{2}\right]
$$

where

$$
\begin{gathered}
C_{r}=\pi^{-3 / 2}(1+2 K)^{3 / 8}, \\
c_{r}=(1+2 K)^{1 / 2}-1 \\
C_{p}=\pi^{-3 / 2}(1+2 K)^{-3 / 8}, \\
c_{p 1}=\frac{(1+2 K)^{1 / 2}+1}{4(1+2 K)^{1 / 2}}, \\
c_{p 2}=\frac{(1+2 K)^{1 / 2}-1}{2(1+2 K)^{1 / 2}} .
\end{gathered}
$$

After elementary calculations we arrive at

$\left.\bar{g}(t ; a, b)=\frac{4}{\pi^{1 / 2}}\left[\frac{2}{(a-b)^{2}(1+2 K)^{1 / 2}+(a+b)^{2}}\right]^{3 / 2} \exp \left[-\frac{2}{(a-b)^{2}(1+2 K)^{1 / 2}+(a+b)^{2}} t^{2}\right] 33\right)$

From Eqs. (16) and (33) we are led to the result

$$
I_{f(q ; a, b)}=4\left\langle t^{2}\right\rangle_{(a, b)}=3\left[(a-b)^{2}(1+2 K)^{1 / 2}+(a+b)^{2}\right] .
$$

Then we can easily obtain that

$$
I_{f(q ; 1,0)}=4\left\langle t^{2}\right\rangle_{(1,0)}=3\left[1+(1+2 K)^{1 / 2}\right] .
$$

Then we immediatelly see that all new inequalities are satisfied, (in fact are equalities). 


\section{DISCUSSION}

Fisher information measures the 'narrowness' of the distribution function. Eq. (19) gives a simple relation between the 'narrowness' of three generalized pair distribution functions. For given parameters $a$ and $b$ the generalized pair distribution functions $f(q ; a, b)$ and $f(q ; a,-b)$ satisfy Eq. (19) with the same function $f(q ; 1,0)$. For parameters with a given value of the sum $\left(a^{2}+b^{2}\right)$, the sum of Fisher information $I_{f(q ; a, b)}+I_{f(q ; a,-b)}$ is fixed.

A special choice of the parameters gives important physical quantities. Since the fundamental paper of Sears, Parr and Dinur [25] it is known that there exists a relationship between the quantum mechanical kinetic energy functional and the Fisher information [1]. Here, we point out that the Fisher information can be decomposed into two parts corresponding to the decomposition of the kinetic energy. Koga [22] showed that for $a=b=1$ Eq. (19) gives a decomposition of the kinetic energy $T$ into relative (intracule) $T_{\text {int }}$ and center of mass (extracule) $T_{\text {ext }}$ kinetic energies:

$$
T=T_{\text {int }}+T_{\text {ext }}
$$

where

$$
T_{\text {int }}=\frac{N}{8}\left\langle p_{\text {int }}^{2}\right\rangle
$$

and

$$
T_{\text {ext }}=\frac{N}{2}\left\langle p_{\text {ext }}^{2}\right\rangle
$$

$p_{\text {int }}$ and $p_{\text {ext }}$ are the intracule and extracule radii in the momentum space. Note that Koga normalized the generalized pair functions to $N(N-1) / 2$ and therefore obtained different factors in Eqs.(37) and (38). The center of mass momentum is defined with a factor 2 in the denominator leading to a factor $N / 2$ in Eq.(38).

From Eqs. (14) and (36)- (38) we obtain

$$
\begin{gathered}
T \geq \frac{N}{8} I_{f(q ; 1,0)}, \\
T_{\text {int }} \geq \frac{N}{8} I_{f(q ; 1,-1)}
\end{gathered}
$$

and

$$
T_{e x t} \geq \frac{N}{8} I_{f(q ; 1,1)}
$$


In the Moshinsky model

$$
\begin{gathered}
\left\langle t^{2}\right\rangle_{(1,1)}=3, \\
\left\langle t^{2}\right\rangle_{(1,-1)}=3(1+2 K)^{1 / 2}
\end{gathered}
$$

and

$$
\left\langle t^{2}\right\rangle_{(1,0)}=\frac{3}{4}\left[1+(1+2 K)^{1 / 2}\right]=T_{0}=\frac{1}{2} E_{0}
$$

where $E_{0}$ and $T_{0}$ are the ground-state total and kinetic energies, respectively.

To summarize, the major result of this paper is to establish a relationship between three measures of Fisher information constructed from generalized pair density functions. It finds an application in the decomposition of the kinetic energy. Results are illustrated by the Moshinsky model.

[1] R. A. Fisher, Proc. Cambridge Philos. Soc.22, 700 (1925).

[2] B. R. Frieden, Am. J. Phys. 57, 1004 (1989).

[3] M. Reginatto, Phys. Rev. A 58, 1775 (1998).

[4] B. R. Frieden Physics from Fisher Information. A unification. (Cambridge U. P., 1998).

[5] R. Nalewajski, Chem. Phys. Lett. 372, 28 (2003).

[6] Á. Nagy, J. Chem. Phys. 119, 9401 (2003).

[7] E. Romera, P. Sánchez-Moreno and J. S. Dehesa, Chem. Phys. Lett. 414468 (2005).

[8] Á. Nagy, Chem. Phys. Lett.425, 157 (2006).

[9] Á. Nagy and K. D. Sen, Phys. Lett. A 360, 291 (2006).

[10] I. Hornyák and Á. Nagy, Chem. Phys. Lett. 437, 132 (2007).

[11] E. Romera and J. S. Dehesa, J. Chem. Phys. 120, 8906 (2004).

[12] E. Romera, Mol. Phys. 100, 3325 (2002).

[13] Á. Nagy and K. D. Sen, Phys. Lett. A 360, 291 (2006).

[14] S. B. Liu, J. Chem. Phys. 126, 191107 (2007).

[15] Á. Nagy, Chem. Phys. Lett. 449, 212 (2007).

[16] Á. Nagy and S. B. Liu, Phys. Lett. A 3721654 (2008). 
[17] J. B. Szabó, K. D. Sen and Á. Nagy, Phys. Lett. A 3722428 (2008).

[18] S. Lopez-Rosa,R.O. Esquivel, J.C. Angulo,J. Antolin, J.S. Dehesa, and N. Flores-Gallegos, J. Chem. Theor. Compt. 6, 145 (2010).

[19] I.P. Hamilton and R. A. Mosna, J. Comp. Appl. Math. 233, 1542 (2010).

[20] R.F. Nalewajski, Int. J. Quantum Chem. 108, 2230 (2008)

[21] S.B. Liu, J. Chem. Phys. 126, 244193 (2007).

[22] T. Koga, J. Chem. Phys. 114, 2511 (2001).

[23] M. W. Hall, Phys. Rev. A 62, 012107 (2000).

[24] A. J. Stam, Inf. Control 2, 101 (1959).

[25] S. B. Sears, R. G. Parr and U. Dinur, Israel J. Chem. 19, 165 (1980).

\section{Acknowledgements}

ÁN acknowledges grant OTKA No. T67923. ER acknowledges the Spanish projects FQM-165/0207 and FIS2008-01143. 